\title{
Models for low-energy Lorentz violation in the photon sector: Addendum to 'Consistency of isotropic modified Maxwell theory'
}

\author{
F.R. Klinkhamer*, M. Schreck \\ Institute for Theoretical Physics, University of Karlsruhe, \\ Karlsruhe Institute of Technology, 76128 Karlsruhe, Germany
}

\begin{abstract}
In a previous article [1], we established the consistency of isotropic modified Maxwell theory for a finite range of the Lorentz-violating parameter $\widetilde{\kappa}_{\text {tr }}$, which includes both positive and negative values of $\widetilde{\kappa}_{\mathrm{tr}}$. As an aside, we mentioned the existence of a physical model which, for low-energy photons, gives rise to isotropic modified Maxwell theory with a positive parameter $\widetilde{\kappa}_{\text {tr }}$ (corresponding to a "slow" photon). Here, we present a related model which gives rise to isotropic modified Maxwell theory with a negative parameter $\widetilde{\kappa}_{\mathrm{tr}}$ (corresponding to a "fast" photon). Both models have an identical particle content, photon and Dirac particles, but differ in the type of spacetime manifold considered.
\end{abstract}

Key words: Lorentz violation, spacetime topology, quantum fields in curved spacetime PACS: 11.30.Cp, 04.20.Gz, 04.62.+v

We have investigated the consistency of isotropic modified Maxwell theory in Ref. [1], which contains an extensive list of references. The photon $(\gamma)$ of this theory has the following dispersion law and velocity:

$$
\begin{aligned}
\omega_{\gamma}(\mathbf{k}) & \equiv c\left|k^{0}(\mathbf{k})\right|=\sqrt{\frac{1-\widetilde{\kappa}_{\mathrm{tr}}}{1+\widetilde{\kappa}_{\mathrm{tr}}}} c|\mathbf{k}|=\left(1-\widetilde{\kappa}_{\mathrm{tr}}+\mathrm{O}\left(\widetilde{\kappa}_{\mathrm{tr}}^{2}\right)\right) c|\mathbf{k}|, \\
v_{\gamma, \text { group }}(\mathbf{k}) & \equiv\left|\frac{\partial \omega_{\gamma}(\mathbf{k})}{\partial \mathbf{k}}\right|=\sqrt{\frac{1-\widetilde{\kappa}_{\mathrm{tr}}}{1+\widetilde{\kappa}_{\mathrm{tr}}}} c=\left(1-\widetilde{\kappa}_{\mathrm{tr}}+\mathrm{O}\left(\widetilde{\kappa}_{\mathrm{tr}}^{2}\right)\right) c .
\end{aligned}
$$

${ }^{*}$ Corresponding Author

Email addresses: frans.klinkhamer@kit.edu (F.R. Klinkhamer), marco.schreck@kit.edu (M. Schreck) 
Here, $\hbar \mathbf{k}$ is the three-momentum of the photon, $\hbar \omega_{\gamma}$ the energy of the photon, $\widetilde{\kappa}_{\text {tr }}$ a dimensionless model parameter (coupling constant) characterizing the strength of the Lorentz violation, and $c$ the velocity of light in vacuum without Lorentz violation (a better definition will be given shortly). The main result of Ref. [1] was that isotropic modified Maxwell theory is consistent (i.e., causal and unitary) for model parameters $\widetilde{\kappa}_{\operatorname{tr}} \in(-1,1]$.

The question arises if there exists an underlying physical model which, at low photon energies, gives rise to an effective theory containing isotropic modified Maxwell theory. In Ref. [1], we have briefly discussed a model [2] which gives isotropic modified Maxwell theory with $\widetilde{\kappa}_{\text {tr }}>0$ (corresponding to a "slow" photon with velocity below $c$ ). Here, we present another model which gives isotropic modified Maxwell theory with $\widetilde{\kappa}_{\text {tr }}<0$ (corresponding to a "fast" photon with velocity above $c$ ).

Both models have the same particle content but different spacetime manifolds. The particle theory is, in fact, a trivial extension of standard quantum electrodynamics (QED) with photons, charged Dirac particles ("electrons"), and uncharged Dirac particles ("neutrinos") propagating in a background spacetime. There are thus three quantum fields with the following physical charges and masses of the corresponding particles:

$$
\begin{array}{ll}
A_{\mu}(x): Q=0, & M=0, \\
\psi_{e}(x): Q=-|e|, & M=m_{e}, \\
\psi_{\nu}(x): Q=0, & M=m_{\nu},
\end{array}
$$

to which is added the classical background field $g_{\mu \nu}(x)$. This relatively simple theory allows for a clean operational definition of $c$, namely, as the maximal attainable velocity of the neutrino in a local orthonormal frame:

$$
\begin{aligned}
c & \equiv\left[\lim _{|\mathbf{k}| \rightarrow \infty} \frac{\omega_{\nu}(\mathbf{k})}{|\mathbf{k}|}\right]^{(\text {local })}, \\
\left(\omega_{\nu}(\mathbf{k})\right)^{2} & =c^{2}|\mathbf{k}|^{2}+\left(m_{\nu} c^{2} / \hbar\right)^{2} .
\end{aligned}
$$

Even without weak interactions, it is still possible, in principle, to monitor the propagation of a neutrino wave packet by its gravitational effects on test masses. 
The first model, now, considers these quantum fields to propagate over a certain type of classical spacetime-foam manifold, which is flat over large scales but has small localized defects ("holes"). The propagation of standard electromagnetic waves in different realizations of such a spacetime foam has been investigated in Ref. [2]. The result is a modified photon dispersion law in the long-wavelength limit, which can be compared to the dispersion law (1a) of isotropic modified Maxwell theory. The parametric dependence of the effective Lorentz-violating coupling constant $\widetilde{\kappa}_{\text {tr }}$ turns out to be as follows:

$$
\widetilde{\kappa}_{\operatorname{tr}} \sim(b / l)^{4}
$$

with $b$ the typical size of a spacetime defect and $l$ the mean separation. The calculation [2] assumes $l \gg b$ and uses the long-wavelength limit $\lambda_{\text {photon }} \gg l$.

Physically, a positive Lorentz-violating parameter $\widetilde{\kappa}_{\mathrm{tr}}$ can thus be interpreted as the spacetime-volume fraction excluded by defects 1 . An equally important result from Ref. [2] is that the dispersion law of Dirac fermions is not modified to leading order. Hence, the model neutrino propagating over this type of classical spacetime foam keeps the standard dispersion law (3b), and similarly for the model electron. The effective theory from this first model thus corresponds to the isotropic modified Maxwell theory with $\widetilde{\kappa}_{\text {tr }}>0$ and the standard Dirac theory for the fermions. Concretely, the model has an effective photon velocity which is smaller than the maximum velocity $c$ of the neutrino and electron.

The second model considers the same quantum fields to propagate over a smooth simplyconnected spacetime manifold with large-scale curvature. Drummond and Hathrell [4] (see also Ref. [5] for further discussion) have already examined the propagation of photons in a gravitational background, taking into account the effects of vacuum polarization by the

\footnotetext{
${ }^{1} \mathrm{~A}$ spacetime manifold with punctures $(b \rightarrow 0$ for fixed $l$ ) may still give a finite positive value for the effective coupling constant $\widetilde{\kappa}_{\text {tr }}$. The particle content then needs to be changed to that of a chiral gauge theory, having, for example, a $U(1)$ gauge boson with massless Weyl fermions in a "complex" representation (appropriate charges proportional to $e$ ). Anomalous effects then give a positive parameter $\widetilde{\kappa}_{\text {tr }} \propto\left(e^{2} / 4 \pi\right)^{2} \equiv$ $\alpha^{2}$, according to Eqs. (5.26)-(5.28) of Ref. [3]. Incidentally, this quadratic dependence on $\alpha$ corrects the erroneous statement in Footnote. 4 of Ref. [1] about a linear dependence.
} 
electron/positron field. Again, the calculation uses a long-wavelength limit (see below). Notably for a Friedmann-Robertson-Walker (FRW) universe with nonvanishing matter content (energy density $\rho$ and pressure $P$ ), they found that photons propagate with a speed that is both isotropic and larger than the speed of light in flat Minkowski spacetime. Translated to a model with particle content (2), the result is that photons propagate in FRW spacetime with a speed that is both isotropic and larger than the maximum attainable speed of the uncharged Dirac particle (neutrino). Conceptually, it is, of course, better to compare velocities in the same spacetime.

The second model with an FRW background metric thus gives rise to an effective theory [4] with the linearized photon dispersion law (1a) of isotropic modified Maxwell theory for negative $\widetilde{\kappa}_{\text {tr }}$. Reinstating $\hbar$ and $c$ in Eqs. (2.19) and (6.8) of Ref. [4] gives

$$
\frac{\left|k^{0}\right|}{|\mathbf{k}|}=1+\frac{11}{45} \alpha\left(\frac{\hbar}{c^{3}}\right)^{2} \frac{1}{\left(m_{e}\right)^{2}} G_{N}[\rho(t)+P(t)]
$$

with fine-structure constant $\alpha \equiv e^{2} /(4 \pi \hbar c)$ and cosmic time $t$ from the FRW metric. The velocity $c$ in (5) corresponds to the maximum neutrino velocity (3a), as will be discussed further below.

In order to get an estimate for the magnitude of this result, consider the cold-darkmatter component of the present (spatially-flat) universe with pressure $P=P_{\mathrm{CDM}}=0$ and an energy density $\rho=\rho_{\mathrm{CDM}}$ equal to approximately one quarter of the critical energy density $\rho_{c} \equiv 3 c^{2} H_{0}^{2} /\left(8 \pi G_{N}\right)$ for Hubble constant $H_{0}$. With these approximations, (5) becomes

$$
\left[\frac{\left|k^{0}\right|}{|\mathbf{k}|}\right]^{\text {(present universe) }} \simeq 1+\frac{11}{180} \alpha\left(\frac{\hbar}{m_{e} c}\right)^{2} \frac{G_{N} \rho_{c}}{c^{4}} \simeq 1+\frac{11}{480 \pi} \alpha\left(\frac{\hbar}{m_{e} c}\right)^{2} \frac{H_{0}^{2}}{c^{2}} .
$$

The parametric dependence of the corresponding Lorentz-violating parameter $\widetilde{\kappa}_{\mathrm{tr}}$ can then be written as follows [4]:

$$
\begin{aligned}
\widetilde{\kappa}_{\mathrm{tr}} & \sim-\alpha\left(\lambda_{e, \text { Compton }} / L_{\text {Hubble }}\right)^{2}, \\
\lambda_{e, \text { Compton }} & \equiv \hbar /\left(m_{e} c\right), \quad L_{\text {Hubble }} \equiv c / H_{0} .
\end{aligned}
$$

Hence, we have expressed the effective Lorentz-violating parameter by the fundamental length scales that play a role in this setup: the reduced Compton wavelength of the electron and the Hubble length, which is the curvature scale of the cosmological background 
spacetime. 2 As mentioned above, the calculation of Ref. [4] uses the long-wavelength limit, specifically $\lambda_{\text {photon }} \gg \lambda_{e \text {, Compton }}$, but the calculation still keeps $\lambda_{\text {photon }} \ll L_{\text {Hubble }}$.

The fine structure constant appears in (5)-(7) since the effective Lorentz violation results from a one-loop quantum effect. The electron contributes to the quantum fluctuation of the photon and gives it a size of the order of the electron Compton wavelength. Since the photon acquires a size by quantum fluctuations, the equivalence principle is not exactly valid for this particle, which is no longer pointlike. The same holds for the electron but not for the neutrino (at least, the neutrino of the model considered, with a photon but not a $Z$ boson).

The effective theory from this second model thus corresponds to isotropic modified Maxwell theory with $\widetilde{\kappa}_{\text {tr }}<0$ and standard Dirac theory for the neutrino. Concretely, the model has an effective photon velocity which is larger than the maximum velocity $c$ of the neutrino 3

It is clear that both models can be combined: the QED fields (2) now propagate over a spacetime manifold with both small-scale structure and large-scale curvature. Depending on the relative strength of (44) and (7a), the effective photon velocity will then be below or above the maximum neutrino velocity $c$.

To summarize, there exist underlying physical models which, in the long-wavelength limit of the photon, give rise to isotropic modified Maxwell theory with both $\widetilde{\kappa}_{\text {tr }} \geq 0$ and $\widetilde{\kappa}_{\text {tr }} \leq 0$. But these models generate the isotropic-modified-Maxwell dispersion law only to linear order in $\widetilde{\kappa}_{\text {tr }}$. In other words, isotropic modified Maxwell theory is obtained for sufficiently small values of $\left|\widetilde{\kappa}_{\mathrm{tr}}\right|$. This may explain the consistency of isotropic modified Maxwell theory for small values of the Lorentz-violating parameter. The consistency for finite parameter values $\widetilde{\kappa}_{\text {tr }} \in(-1,1]$ is, therefore, a nontrivial result [1].

\footnotetext{
${ }^{2}$ In the present universe with $H_{0} \sim 70 \mathrm{~km} \mathrm{~s}^{-1} \mathrm{Mpc}^{-1}, L_{\text {Hubble }} \sim 10^{26} \mathrm{~m}, \alpha \sim 10^{-2}$, and $m_{e} \sim 0.5 \mathrm{MeV}$, the numerical value of (7a) is of order $-10^{-79}$, which may be compared to the current experimental bound at the $-10^{-15}$ level [6].

${ }^{3}$ To leading order in $\alpha$, there is, however, no Lorentz violation for a photon propagating in a de Sitter background [4, [5], giving $\widetilde{\kappa}_{\mathrm{tr}}=0$ in the effective modified Maxwell theory. This result is consistent with (5), because the corresponding Lorentz-invariant vacuum energy and pressure are related by $\rho_{V}+P_{V}=0$.
} 


\section{References}

[1] F.R. Klinkhamer, M. Schreck, "Consistency of isotropic modified Maxwell theory: Microcausality and unitarity," Nucl. Phys. B 848 (2011) 90, arXiv:1011.4258.

[2] S. Bernadotte, F.R. Klinkhamer, "Bounds on length scales of classical spacetime foam models," Phys. Rev. D 75 (2007) 024028, arXiv:hep-ph/0610216.

[3] F.R. Klinkhamer and C. Rupp, "Spacetime foam, CPT anomaly, and photon propagation," Phys. Rev. D 70 (2004) 045020, arXiv:hep-th/0312032.

[4] I.T. Drummond, S.J. Hathrell, "QED vacuum polarization in a background gravitational field and its effect on the velocity of photons," Phys. Rev. D 22 (1980) 343.

[5] G.M. Shore, "Accelerating photons with gravitational radiation," Nucl. Phys. B 605 (2001) 455, arXiv:gr-qc/0012063

[6] F.R. Klinkhamer, M. Schreck, "New two-sided bound on the isotropic Lorentz-violating parameter of modified Maxwell theory," Phys. Rev. D 78 (2008) 085026, arXiv:0809.3217. 